\title{
Avaliação da resistência ao cisalhamento de resinas compostas flow autoadesivos versus convencionais: uma revisão sistemática
}

\author{
Shear strength evaluation of self-adhesive versus conventional flow composites resins: a systematic \\ review
}

Evaluación de la resistencia al cizallamiento de materiales compuestos autoadhesivos frente a compuestos convencionales de flujo: una revisión sistemática

Recebido: 10/01/2022 | Revisado: 14/01/2022 | Aceito: 21/01/2022 | Publicado: 23/01/2022

Cassia Thaís Iurkiv Zanatta

ORCID: https://orcid.org/0000-0003-3300-4350 Universidade Estadual do Oeste do Paraná, Brasil E-mail: cassiaiurkiv@gmail.com

Stéphane Vanelli da Silva

ORCID: https://orcid.org/0000-0002-9672-1181 Universidade Estadual do Oeste do Paraná, Brasi E-mail: stephanevaneli@ hotmail.com

Stephanie Bonamin

ORCID: https://orcid.org/0000-0002-4386-6838 Universidade Estadual do Oeste do Paraná, Brasil E-mail: stebonamin@gmail.com

Veridiana Camilotti

ORCID: https://orcid.org/0000-0002-3004-3939

Universidade Estadual do Oeste do Paraná, Brasi

E-mail: vericamilotti@hotmail.com

Márcio José Mendonça

ORCID: https://orcid.org/0000-0002-6953-7135 Universidade Estadual do Oeste do Paraná, Brasil

E-mail: dr.mendonca@uol.com.br

Julio Katuhide Ueda

ORCID: https://orcid.org/0000-0002-8664-942X

Universidade Estadual do Oeste do Paraná, Brasil

E-mail: julioueda2020@gmail.com

\begin{abstract}
Resumo
Pouco se sabe sobre as resinas compostas fluidas autoadesivas, uma vez que a maior parte da literatura menciona as resinas compostas fluidas convencionais. Então, o objetivo do presente trabalho foi revisar exclusivamente o comportamento da adesão ao esmalte e à dentina das resinas compostas fluidas autoadesivas em comparação com a resina composta fluida convencional. Uma pesquisa bibliográfica foi realizada de 2010 a 2021 na base de dados Pubmed. O termo de busca utilizado foi "self-adhering flowable composite", onde 61 artigos completos foram avaliados e somente 11 foram selecionados. Foram elegíveis para a seleção apenas estudos que avaliaram a resistência de união pelo teste de cisalhamento ou microcisalhamento da resina composta fluida autoadesiva aplicada na estrutura dental. Com base nessa revisão sistemática, conclui-se que a resina fluida autoadesiva demonstrou valores de resistência de união ao esmalte e à dentina inferior que a resina fluida convencional. A associação do sistema adesivo de condicionamento ácido total com resina fluida autoadesiva mostrou valores de resistência de união maior que o uso dos autoadesivos sozinhos. A resina fluida autoadesiva obteve valores de resistência de união similar aos materiais restauradores à base de ionômero de vidro.
\end{abstract}

Palavras-chave: Resinas compostas; Resistência ao cisalhamento; Revisão sistemática.

\begin{abstract}
Little is known about self-adhesive flowable composite resins since most of the literature mentions conventional flowable composite resins. Therefore, the present work aimed to exclusively review the bonding behavior to enamel and dentin of self-adhesive flowable composite resins compared to conventional flowable composite resin. A literature search was carried out from 2010 to 2021 in the Pubmed database. The search term used was "self-adhering flowable composite", where 61 complete articles were evaluated and only 11 were selected. Only studies that evaluated the bond strength by the shear or micro shear test of the self-adhesive fluid composite resin applied to the tooth structure were eligible for selection. Based on this systematic review, it is concluded that the self-adhesive
\end{abstract}


flowable resin demonstrated lower bond strength values to enamel and dentin than conventional flowable resin. The combination of the total acid etch adhesive system with self-adhesive fluid resin showed higher bond strength values than the use of self-adhesives alone. The self-adhesive fluid resin obtained bond strength values similar to glass ionomer-based restorative materials.

Keywords: Composite resins; Shear strength; Systematic review.

\section{Resumen}

Poco se sabe acerca de las resinas compuestas fluidas autoadhesivas, ya que la mayor parte de la literatura menciona resinas compuestas fluidas convencionales. Por lo tanto, el objetivo del presente trabajo fue revisar exclusivamente el comportamiento de unión al esmalte y la dentina de las resinas compuestas fluidas autoadhesivas en comparación con la resina compuesta fluida convencional. Se realizó una búsqueda bibliográfica de 2010 a 2021 en la base de datos Pubmed. El término de búsqueda utilizado fue "self-adhering flowable composite", donde se evaluaron 61 artículos completos y solo 11 fueron seleccionados. Solo fueron elegibles para la selección los estudios que evaluaron la resistencia de la unión mediante la prueba de cizallamiento o microcizallamiento de la resina compuesta fluida autoadhesiva aplicada a la estructura dental. Con base en esta revisión sistemática, se concluye que la resina fluida autoadhesiva demostró valores más bajos de fuerza de unión al esmalte y la dentina que la resina fluida convencional. La combinación del sistema adhesivo de grabado ácido total con resina fluida autoadhesiva mostró mayores valores de fuerza de unión que el uso de autoadhesivos solos. La resina fluida autoadhesiva obtuvo valores de fuerza de unión similares a los materiales restauradores a base de ionómero de vidrio.

Palabras clave: Resinas compuestas; Resistencia al corte; Revisión sistemática.

\section{Introdução}

O uso das resinas compostas flow ou fluidas se tornou muito comum nas clínicas odontológicas (Abdelraouf et al., 2019; ElEmbaby et al., 2021; Naga et al., 2015; Oz et al., 2020). Inicialmente desenvolvidas para uso em cavidades de classe V (Bayne et al., 1998; Jordehi et al., 2019), hoje em dia as resinas compostas fluidas são utilizadas em diferentes procedimentos clínicos, incluindo a restauração de pequenas lesões de cárie, selamento de cicatrículas e fissuras e, ainda, como forramento para restaurações de resina composta (Oz et al., 2020; Unterbrink \& Liebenberg, 1999). São facilmente inseridas em pequenas cavidades e espera-se que exibam melhor adaptação às paredes da cavidade quando comparadas com as resinas compostas de maior viscosidade (Baroudi et al., 2007; Ikeda et al., 2009; Oz et al., 2020). Devido ao menor conteúdo de carga, es sas resinas compostas têm menor módulo de elasticidade e maior contração de polimerização em comparação com a resina composta convencional (Labella et al., 1999).

Um dos recentes avanços na odontologia é a introdução de resinas compostas fluidas autoadesivas, que são um produto da combinação de dois grupos funcionais: o sistema adesivo de passo único (all-in-one) e a resina composta fluida (ElEmbaby et al., 2021; Garcia et al., 2014; Oz et al., 2020; Vichi et al., 2013). Pela incorporação do monômero funcional glicerofosfato dimetacrilato (GPDM) na composição química de uma dessas resinas compostas fluidas autoadesivas, as etapas dos procedimentos restauradores diretos foram simplificadas (Jordehi et al., 2019; Poss, 2010). Com base nas alegações do fabricante, esse monômero exibe propriedades ácidas, condiciona a estrutura do dente, se une ao cálcio da estrutura do dente e possui dois grupos funcionais de metacrilato, que podem copolimerizar com outros monômeros de metacrilato (Yuan et al., 2015). Portanto, o uso dessas resinas compostas, resulta na redução do tempo necessário para sua aplicação e os erros clínicos e a sensibilidade da técnica diminuem. Contrariamente, foi relatado que o GPDM "condiciona" ao invés de "se unir" à hidroxiapatita (Yoshida et al., 2004). Ainda, com base em estudos anteriores, essas resinas compostas mostram um módulo de elasticidade, dureza (Salerno et al., 2011) e grau de conversão (Czasch \& Ilie, 2013) maior que outras resinas compostas fluidas convencionais. Além disso, essas resinas compostas exibiram maior expansão higroscópica (Wei et al., 2011b) e absorção de água (Wei et al., 2011a) quando comparadas com outras resinas compostas fluidas, 150 dias após a imersão em água.

O padrão ouro de adesão ainda é a forma tradicional onde é realizado o condicionamento total, lavagem e secagem do substrato (van Meerbeek et al., 2020). A abordagem de múltiplos passos de condicionamento ácido total, ao contrário do 
autocondicionamento, envolve uma etapa de condicionamento com ácido fosfórico que cria porosidades na hidroxiapatita do esmalte e expõe as fibras colágenas da dentina. As projeções de resina incorporadas nessas estruturas dentárias condicionadas com ácido promovem uma adesão satisfatória com a superfície do esmalte e dentina, selando efetivamente as margens das restaurações (Abdelraouf et al., 2019; Sachdeva et al., 2016).

Dada a importância de uma adesão efetiva das restaurações de resina composta e considerando a recente introdução de resinas compostas fluidas autoadesivas, a existência de dados limitados sobre o seu desempenho e a controvérsia em relação a sua adesão à estrutura dentária, este estudo teve como objetivo revisar sistematicamente o comportamento da adesão no esmalte e na dentina das resinas compostas fluidas autoadesivas em comparação com a resina composta fluida convencional.

\section{Metodologia}

(i) Critérios de elegibilidade: Elegíveis para inclusão nesta revisão foram os artigos publicados em inglês, datados de 2010 a 2021. Inicialmente os artigos selecionados deveriam incluir os termos de busca no título ou no resumo. Posteriormente os "abstracts" foram lidos e avaliados. Artigos de texto completo e revisões de literatura foram preferidos. Foram elegíveis para a seleção apenas estudos que avaliaram a resistência de união pelo teste de cisalhamento ou microcisalhamento da resina composta fluida autoadesiva aplicada no esmalte e na dentina.

(ii) Fontes de dados: Uma pesquisa bibliográfica completa foi feita na base de dados PubMed Os termos de busca utilizados foram "self"; "adhering"; "flowable" e "composite". A conjunção aditiva "and" (no inglês) foi acrescentada para fornecer o sentido de relação entre os termos.

(iii) Estratégia de Busca: Um total de 61 artigos completos foram triados e avaliados.

(iv) Extração de dados: Apenas artigos relevantes que atenderam aos objetivos da revisão (ou seja, estudos que avaliaram a resistência de união pelo teste de cisalhamento ou microcisalhamento da resina composta fluida autoadesiva aplicada no esmalte e na dentina) foram consideradas. A Figura 1 é um fluxograma que resume o processo de seleção dos artigos. Após os artigos serem examinados pelos títulos e resumos, 50 estudos foram excluídos por não atenderem aos critérios de elegibilidade. Um total de 11 estudos preencheram todos os critérios de seleção e foram incluídos nesta revisão.

Figura 1: Fluxograma de pesquisa dos artigos

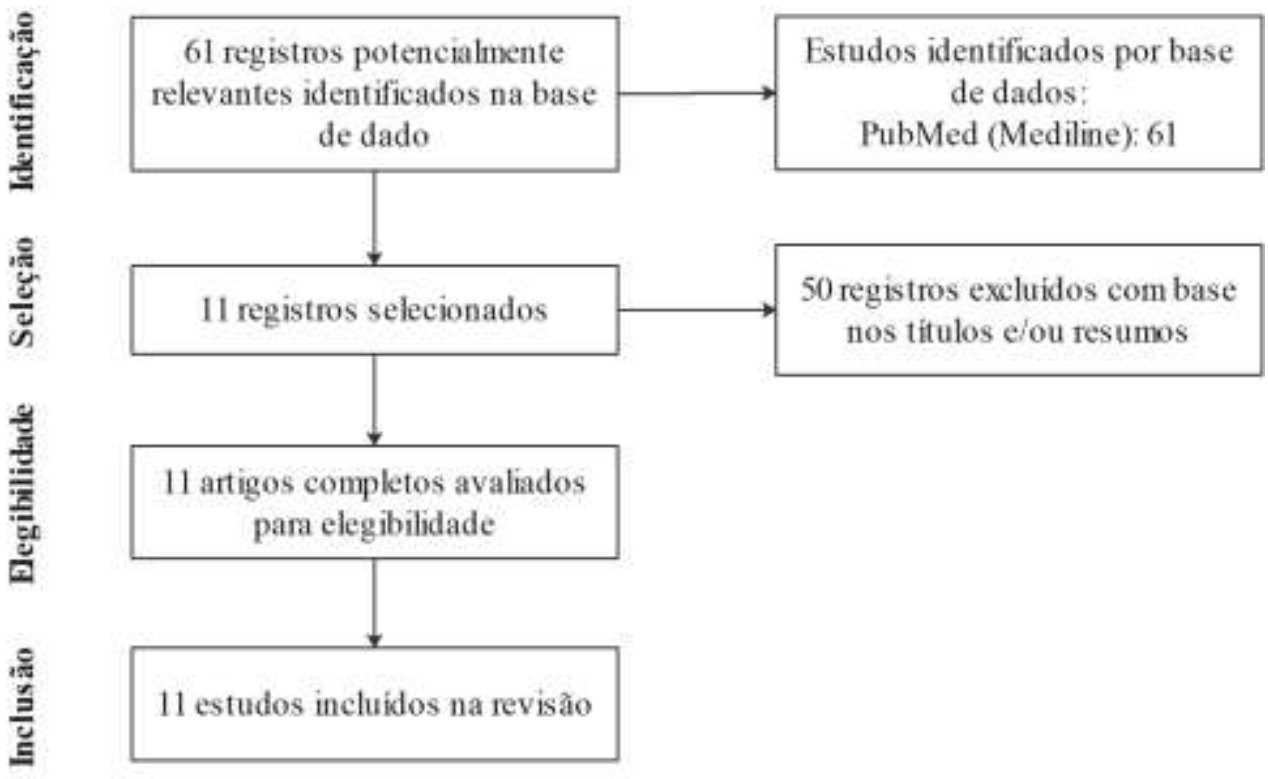




\section{Resultados}

Um total de 61 registros potencialmente relevantes foram identificados na base de dados e examinados pelos títulos e resumos, dos quais 50 foram excluídos por não atenderem aos critérios de elegibilidade. Um total de 11 estudos preencheram os critérios de seleção e foram incluídos nesta revisão (Tabela 1).

Tabela 1 - Dados dos estudos incluídos na revisão.

\begin{tabular}{|c|c|c|c|c|c|c|c|}
\hline Estudo & Ano & País & $\begin{array}{l}N^{\circ} \text { de } \\
\text { dentes } \\
\text { (por } \\
\text { grupo) }\end{array}$ & $\begin{array}{l}\text { Tipo de } \\
\text { dente }\end{array}$ & Objetivo & Material usado & Tipo de material \\
\hline $\begin{array}{l}\text { Abdelraouf } \\
\text { (Abdelraouf et } \\
\text { al., 2019) }\end{array}$ & 2019 & Egito & 6 & $\begin{array}{l}\text { Molar } \\
\text { permanente }\end{array}$ & $\begin{array}{l}\text { SBS* no } \\
\text { esmalte e } \\
\text { dentina }\end{array}$ & $\begin{array}{ll} & \text { Dyad-flow (Kerr, USA); } \\
\text { - } & \text { Filtek }^{\mathrm{TM} Z 350-X T} \text { (3MESPE, USA) }\end{array}$ & $\begin{array}{l}\text { - } \\
\text { Resina fluida } \\
\text { autoadesiva } \\
\text { Resina fluida } \\
\text { convencional }\end{array}$ \\
\hline $\begin{array}{l}\text { Bektas (Bektas } \\
\text { et al., 2013) }\end{array}$ & 2013 & Turquia & 10 & $\begin{array}{l}\text { Molar } \\
\text { permanente }\end{array}$ & $\begin{array}{l}\mu \mathrm{SBS}^{* *} \text { na } \\
\text { dentina }\end{array}$ & $\begin{array}{ll}\text { Vertise Flow (Kerr Corporation, } \\
\text { Orange, CA) } \\
\text { Revolution Formula2 (Kerr } \\
\text { Corporation, Orange, CA) }\end{array}$ & $\begin{array}{l}\text { Resina fluida } \\
\text { autoadesiva } \\
\text { - } \quad \text { Resina fluida } \\
\text { convencional }\end{array}$ \\
\hline $\begin{array}{l}\text { Bumrungruan } \\
\text { (Bumrungruan } \\
\text { \& } \\
\text { Sakoolnamarka, } \\
\text { 2016) }\end{array}$ & 2016 & Tailândia & 20 & $\begin{array}{l}\text { Molar } \\
\text { permanente }\end{array}$ & $\begin{array}{l}\mu \text { SBS na } \\
\text { dentina }\end{array}$ & $\begin{array}{l}\text { Vertise Flow (Kerr Corporation, } \\
\text { Orange, CA) } \\
\text { Premise flowable (Kerr Corporation) }\end{array}$ & $\begin{array}{l}\text { - } \quad \text { Resina fluida } \\
\text { autoadesiva } \\
\text { - } \quad \text { Resina fluida } \\
\text { convencional }\end{array}$ \\
\hline $\begin{array}{l}\text { Durmuşlar } \\
\text { (Durmuşlar \& } \\
\text { Ölmez, 2017) }\end{array}$ & 2017 & Turquia & 20 & $\begin{array}{l}\text { Molar } \\
\text { decíduo }\end{array}$ & $\begin{array}{l}\mu \mathrm{TBS} * * * \text { na } \\
\text { dentina }\end{array}$ & $\begin{array}{l}\text { Vertise Flow (Kerr Corporation, } \\
\text { Orange, CA) } \\
\text { G-aenial Universal Flo }{ }^{\circledR}(\mathrm{GC} \\
\text { Corporation, Tokyo, JAPAN) } \\
\text { Tetric }{ }^{\circledR} \text { N-flow (Ivoclar Vivadent, } \\
\text { Schaan, Liechtenstein) }\end{array}$ & $\begin{array}{l}\text { Resina fluida } \\
\text { autoadesiva } \\
\text { Resina fluida } \\
\text { convencional } \\
\text { Resina fluida } \\
\text { convencional }\end{array}$ \\
\hline $\begin{array}{l}\text { Goracci } \\
\text { (Goracci et al., } \\
\text { 2013) }\end{array}$ & 2013 & Itália & 20 & $\begin{array}{l}\text { Pré-molar } \\
\text { permanente }\end{array}$ & $\begin{array}{l}\text { SBS no } \\
\text { esmalte }\end{array}$ & $\begin{array}{l}\text { Vertise Flow (Kerr Corporation, } \\
\text { Orange, CA) } \\
\text { Transbond XT Paste (3M Unitek, } \\
\text { Monrovia, CA, USA) }\end{array}$ & $\begin{array}{l}\text { Resina fluida } \\
\text { autoadesiva } \\
\text { - } \quad \text { Resina fluida } \\
\text { convencional }\end{array}$ \\
\hline $\begin{array}{l}\text { Pacifici (Pacifici } \\
\text { et al., 2013) }\end{array}$ & 2013 & Itália & 10 & $\begin{array}{l}\text { Molar } \\
\text { decíduo }\end{array}$ & $\begin{array}{l}\text { SBS na } \\
\text { dentina }\end{array}$ & $\begin{array}{ll}\text { - } & \text { Vertise Flow (Kerr Corporation, } \\
\text { Orange, CA) } \\
\text { - } & \text { Premise flowable (Kerr Corporation) } \\
\text { - } & \text { Fuji II LC (GC, Tokyo, Japan) } \\
\text { Fuji IX (GC, Tokyo, Japan) }\end{array}$ & $\begin{array}{ll}\text { - } & \text { Resina fluida } \\
\text { - } & \text { autoadesiva } \\
\text { Resina fluida } \\
\text { convencional } \\
\text { - } & \text { Ionômero de vidro } \\
\text { - } & \text { fotopolimerizável } \\
\text { Ionômero de vidro } \\
\text { convencional }\end{array}$ \\
\hline $\begin{array}{l}\text { Poorzandpoush } \\
\text { (Poorzandpoush } \\
\text { et al., 2019) }\end{array}$ & 2019 & Irã & 16 & $\begin{array}{l}\text { Caninos e } \\
\text { molares } \\
\text { decíduos }\end{array}$ & $\begin{array}{l}\text { SBS na } \\
\text { dentina }\end{array}$ & $\begin{array}{ll}\text { - } & \text { Vertise Flow (Kerr Corporation, } \\
\text { Orange, CA) } \\
\text { - } \quad \text { Premise flowable (Kerr Corporation) } \\
\text { Ionolux (Voco) }\end{array}$ & $\begin{array}{ll}\text { - } & \text { Resina fluida } \\
\text { autoadesiva } \\
\text { - } \\
\text { Resina fluida } \\
\text { convencional } \\
\text { - } \quad \text { Ionômero de vidro } \\
\text { fotopolimerizável }\end{array}$ \\
\hline $\begin{array}{l}\text { Sachdeva } \\
\text { (Sachdeva et al., } \\
\text { 2016) }\end{array}$ & 2016 & Índia & 30 & $\begin{array}{l}\text { Dente } \\
\text { decíduo }\end{array}$ & $\begin{array}{l}\text { SBS na } \\
\text { dentina }\end{array}$ & $\begin{array}{ll}\text { - } & \text { Dyad Flow (Kerr Corporation, } \\
\text { Orange, CA) } \\
\text { Fusio Liquid Dentin (Pentron } \\
\text { Clinical Technologies) } \\
\text { G-aenial Universal Flo (GC) }\end{array}$ & $\begin{array}{ll}\text { - } & \text { Resina fluida } \\
\text { autoadesiva } \\
\text { Resina fluida } \\
\text { autoadesiva } \\
\text { Resina fluida } \\
\text { convencional }\end{array}$ \\
\hline $\begin{array}{l}\text { Shafiei (Shafiei } \\
\text { \& Saadat, 2016) }\end{array}$ & 2016 & Irã & 10 & $\begin{array}{l}\text { Molar } \\
\text { permanente }\end{array}$ & $\begin{array}{l}\text { SBS na } \\
\text { dentina }\end{array}$ & $\begin{array}{l}\text { Vertise Flow (Kerr Corporation, } \\
\text { Orange, CA) }\end{array}$ & $\begin{array}{l}\text { - } \quad \text { Resina fluida } \\
\text { autoadesiva }\end{array}$ \\
\hline $\begin{array}{l}\text { Tuloglu } \\
\text { (Tuloglu et al., } \\
\text { 2014) }\end{array}$ & 2014 & Turquia & 10 & $\begin{array}{l}\text { Molar } \\
\text { permanente } \\
\text { e decíduo }\end{array}$ & $\begin{array}{l}\text { SBS na } \\
\text { dentina }\end{array}$ & $\begin{array}{l}\text { Vertise Flow (Kerr Corporation, } \\
\text { Orange, CA) } \\
\text { Filtek Ultimate Flowable Restorative } \\
\text { (3MESPE, USA) }\end{array}$ & $\begin{array}{l}\text { Resina fluida } \\
\text { autoadesiva } \\
\text { - } \\
\text { Resina fluida } \\
\text { convencional }\end{array}$ \\
\hline $\begin{array}{l}\text { Yuan (Yuan et } \\
\text { al., 2015) }\end{array}$ & 2015 & China & 10 & $\begin{array}{l}\text { Molar } \\
\text { permanente }\end{array}$ & $\begin{array}{l}\mu \text { TBS na } \\
\text { dentina }\end{array}$ & $\begin{array}{l}\text { - Dyad Flow (Kerr, Orange, CA, USA) } \\
\text { Filtek Z350 Flowable (3M ESPE, } \\
\text { USA) }\end{array}$ & $\begin{array}{l}\text { Resina fluida } \\
\text { autoadesiva } \\
\text { Resina fluida } \\
\text { convencional }\end{array}$ \\
\hline
\end{tabular}

* SBS: shear bond strength (resistência ao cisalhamento); $\mu$ SBS**: micro-shear bond strength (resistência ao microcisalhamento; $\mu$ TBS***: micro-tensile bond strength (resistência à microtração) - Fonte: Autores. 


\section{Análise descritiva}

Duas diferentes marcas comerciais de resinas compostas fluidas autoadesivas foram avaliadas nesta revisão (Tabela 2). Dos estudos incluídos, oito avaliaram a resina Vertise Flow (Kerr Corporation, Orange, CA, USA) (Bektas et al., 2013; Bumrungruan \& Sakoolnamarka, 2016; Durmuşlar \& Ölmez, 2017; Goracci et al., 2013; Pacifici et al., 2013; Poorzandpoush et al., 2019; Shafiei \& Saadat, 2016; Tuloglu et al., 2014) e três avaliaram a resistência de união ao esmalte do Fusio Dentin Liquid (Pentron Clinical, Orange, CA, USA) (Abdelraouf et al., 2019; Sachdeva et al., 2016; Yuan et al., 2015)

Tabela 2 - Resinas compostas fluidas autoadesivas avaliadas.

\begin{tabular}{|c|c|c|c|c|}
\hline $\begin{array}{c}\text { Materiais/ } \\
\text { Fabricantes }\end{array}$ & & Descrição & Composição Geral & Instruções de uso \\
\hline $\begin{array}{l}\text { Vertise Flow, } \\
\text { Corporation, } \\
\text { CA) }\end{array}$ & $\begin{array}{l}\text { (Kerr } \\
\text { Orange, }\end{array}$ & $\begin{array}{l}\text { Resina } \\
\text { composta fluida } \\
\text { autoadesiva }\end{array}$ & $\begin{array}{l}\text { GPDM, HEMA, cargas } \\
\text { prepolimerizadas, fluoreto de } \\
\text { itérbio nanométrico, carga de } \\
\text { vidro de bário de } 1 \mu \mathrm{m} \text {, sílica } \\
\text { coloidal nanométrico. }\end{array}$ & $\begin{array}{l}\text { Dispensar Vertise Flow na cavidade com a ponta dispensadora } \\
\text { fornecida. Usar o pincel fornecido para aplicar o Vertise Flow } \\
\text { em toda a parede da cavidade com pressão moderada por } 15- \\
20 \mathrm{~s} \text { para obter uma camada fina }(<0,5 \mathrm{~mm}) \text {. Fotopolimerizar } \\
\text { por } 20 \mathrm{~s} \text {. Depois de revestir a parede da cavidade, construir a } \\
\text { restauração com mais Vertise Flow em incrementos de } 2 \mathrm{~mm} \\
\text { ou menos. Fotopolimerizar cada incremento por } 20 \mathrm{~s} \text {. }\end{array}$ \\
\hline $\begin{array}{l}\text { Fusio Liquid } \\
\text { (Pentron } \\
\text { Orange, USA) }\end{array}$ & $\begin{array}{c}\text { Dentin } \\
\text { Clinical, }\end{array}$ & $\begin{array}{l}\text { Resina } \\
\text { composta fluida } \\
\text { autoadesiva }\end{array}$ & $\begin{array}{l}\text { UDMA, TEGDMA, HEMA, 4- } \\
\text { MET, sílica amorfa nanométrica, } \\
\text { vidro de bário silanizado, aditivos } \\
\text { e sistemas de fotopolimerização. }\end{array}$ & $\begin{array}{l}\text { Dispensar uma camada inicial em uma superfície seca; } \\
\text { Pincelar na superfície por } 15-20 \mathrm{~s} \text { com pressão moderada e } \\
\text { fotopolimerizar por } 20 \mathrm{~s} \text {; Aplicar material adicional da seringa } \\
\text { em incrementos de menos de } 2 \mathrm{~mm} \text { e fotopolimerizar cada } \\
\text { incremento por } 20 \mathrm{~s}\end{array}$ \\
\hline
\end{tabular}

Fonte: Poitevin et al. (2013).

Além disso, a resistência adesiva de diferentes resinas compostas fluidas convencionais foi utilizada como parâmetro de comparação. Três estudos avaliaram a Premise Flowable, Kerr, Orange, CA, USA (resina fluida convencional, total etch)

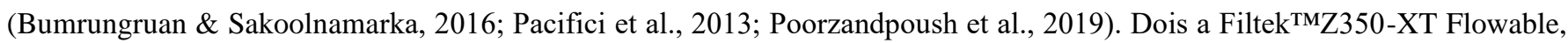
3M-ESPE, USA (resina fluida convencional, total etch) (Abdelraouf et al., 2019; Yuan et al., 2015) e a G-aenial Universal Flo®, GC Corporation, Tokyo, Japan (resina fluida de 1 passo) (Durmuşlar \& Ölmez, 2017; Sachdeva et al., 2016). Resinas fluidas, com uma avaliação cada foram a Filtek Ultimate, 3M/ESPE, St. Paul, MN, USA (resina fluida convencional) (Tuloglu et al., 2014), a Transbond XT Paste, 3M Unitek, Monrovia, CA, USA (resina convencional) (Goracci et al., 2013), a Revolution Formula 2, Kerr Corporation, CA (resina fluida convencional) (Bektas et al., 2013), a Tetric ${ }^{\circledR}$ N-Flow, Ivoclar Vivadent AG, Schaan, Liechtenstein (resina fluida convencional, total-etch) (Durmuşlar \& Ölmez, 2017). Todas as resinas compostas fluidas convencionais foram aplicadas com os seus respectivos sistemas adesivos. Outros materiais avaliados na comparação foram o ionômero de vidro (Fuji II LC e Fuji IX, GC, Tokyo, Japan) (Pacifici et al., 2013) e cimento de ionômero de vidro modificada por resina (Ionolux, Voco, Germany) (Poorzandpoush et al., 2019), com um estudo cada.

\section{Discussão}

O objetivo da presente revisão sistemática foi investigar o comportamento da adesão no esmalte e na dentina da resina composta fluida autoadesiva em comparação com a resina composta fluida convencional.

A revisão demonstrou que as principais resinas fluidas autoadesivas avaliadas eram a Vertise Flow (Kerr Corporation, Orange, CA, USA) (Bektas et al., 2013; Bumrungruan \& Sakoolnamarka, 2016; Durmuşlar \& Ölmez, 2017; Goracci et al., 2013; Pacifici et al., 2013; Poorzandpoush et al., 2019; Shafiei \& Saadat, 2016; Tuloglu et al., 2014) e DyadTM-flow (Kerr, CA, USA) (Abdelraouf et al., 2019; Sachdeva et al., 2016; Yuan et al., 2015), que é a denominação comercial dada para a América Latina; no exterior é comercializada como Fusio Dentin Liquid (Pentron Clinical, Orange, CA, USA) (Miletić, 2018). 
Research, Society and Development, v. 11, n. 2, e20211225576, 2022

(CC BY 4.0) | ISSN 2525-3409 | DOI: http://dx.doi.org/10.33448/rsd-v11i2.25576

Os trabalhos têm mostrado que a resina composta fluida convencional associada a algum tipo de adesivo dentinário, quer seja o etch-and-rinse (Abdelraouf et al., 2019; Bumrungruan \& Sakoolnamarka, 2016; Goracci et al., 2013; Pacifici et al., 2013; Sachdeva et al., 2016; Yuan et al., 2015), autocondicionante (Pacifici et al., 2013) ou universal têm mostrado maiores valores de resistência adesiva em comparação com as resinas compostas fluidas autoadesivas (Pacifici et al., 2013; Sachdeva et al., 2016; Tuloglu et al., 2014). Estes resultados foram semelhantes tanto em estudos laboratoriais de grupos termociclados como não termociclados, como também em estudos clínicos (Bumrungruan \& Sakoolnamarka, 2016). Isto pode ser atribuído ao condicionamento ácido e aplicação do adesivo antes da inserção da resina (Abdelraouf et al., 2019). A alta viscosidade, menor molhabilidade e penetração limitada no substrato dentário da resina fluida autoadesiva também influenciam negativamente na resistência adesiva (Pacifici et al., 2013; Yuan et al., 2015).

No caso do adesivo etch-and-rinse, porosidades superficiais na hidroxiapatita do esmalte são criadas pelo condicionamento com ácido fosfórico e posterior lavagem com água. O adesivo dentinário penetra nessas porosidades, formando os tags de resina, que são os responsáveis pela retenção mecânica e melhor união (Abdelraouf et al., 2019). Já na dentina o ácido fosfórico faz a desmineralização superficial do substrato dentinário, remoção da smear layer e exposição das fibras colágenas. O adesivo penetra nestas fibras colágenas expostas aumentando a retenção micromecânica (Bumrungruan \& Sakoolnamarka, 2016; Poorzandpoush et al., 2019).

Nos adesivos universais um outo fator que pode ajudar no aumento da resistência de união é a interação química à estrutura dentária. Nesses adesivos, que possuem o monômero funcional fosfatado 10 metacriloiloxidecil dihidrogênio fosfato (10-MDP) na sua composição (Abdelraouf et al., 2019; Peumans et al., 2010), ocorre a interação química entre o 10-MDP e a hidroxiapatita, resultando na formação de uma nano-camada estável e mais resistente, o que pode melhorar a resistência de união (Abdelraouf et al., 2019; Yoshida et al., 2012). A deposição de um sal estável "MDP-Ca" acompanhada por uma nanoestratificação podem esclarecer a alta estabilidade da união, comprovada em pesquisas laboratoriais e clínicas (Abdelraouf et al., 2019; Muñoz et al., 2013; Peumans et al., 2010).

Já nos adesivos autocondicionantes como a sua acidez é menor que o ácido fosfórico, produz uma menor desmineralização no substrato dentário e consequentemente uma profundidade de penetração do adesivo mais limitada, levando a uma menor resistência de união (Goracci et al., 2013; Sachdeva et al., 2016)

$\mathrm{O}$ interesse em investigar o comportamento da resina composta fluida autoadesiva reside no fato de ser um material novo, que elimina a necessidade da aplicação prévia de um adesivo dentinário. Tem como vantagem a união química e micromecânica com a estrutura dentária (Pacifici et al., 2013), a redução do tempo clínico e da sensibilidade pós-operatória, bem como a diminuição da complexidade da técnica e do risco de cometer erros durante a sua aplicação, simplificando muito o processo da restauração direta (Bektas et al., 2013). Ainda como característica positiva, apresenta valores de resistência de união comparáveis aos cimentos de ionômero de vidro, sendo uma alternativa para restauração de dentes decíduos (Pacifici et al., 2013). Além disso, a resistência ao desgaste é maior quando comparada aos cimentos de ionômero de vidro e ao ionômero de vidro modificado por resina, com superfície menos porosa, proporcionando uma melhor estética e menor acúmulo de placa bacteriana (Pacifici et al., 2013; Poorzandpoush et al., 2019).

Por serem os primeiros materiais do mercado, as duas resinas compostas fluidas autoadesivas mais citadas na literatura são tecnicamente semelhantes, mas quimicamente usam monômeros ácidos diferentes para estabelecer a autoadesão (Abdelraouf et al., 2019; Bektas et al., 2013; Bumrungruan \& Sakoolnamarka, 2016; Durmuşlar \& Ölmez, 2017; Goracci et al., 2013; Pacifici et al., 2013; Poorzandpoush et al., 2019; Sachdeva et al., 2016; Shafiei \& Saadat, 2016; Tuloglu et al., 2014; Yuan et al., 2015).

A primeira delas é a Fusio Liquid Dentin Dental Flowable Composite (denominado Dyad Flow na América Latina) da Pentron, lançada em maio de 2009. Ela utiliza o monômero funcional 4-META, que tem mostrado uma capacidade de adesão 
química à hidroxiapatita e ao tecido dentário menor ao proporcionado pelo monômero 10-MDP (Yoshida et al., 2004).

A segunda é a Vertise Flow lançado pela Kerr em janeiro de 2010, que utiliza o monômero GPDM ( glicerol fosfato dimetacrilato) (Durmuşlar \& Ölmez, 2017; Eliades et al., 2013; Goracci et al., 2013; Pacifici et al., 2013; Tuloglu et al., 2014). Este GPDM tem uma maior capacidade de estabelecer uma ligação química com a estrutura dentária quando comparado com os ésteres de metacrilato da resina fluida convencional (Goracci et al., 2013). Tem como vantagem possuir um pH de 1,9 que é eficiente na desmineralização do substrato dentário e após a polimerização seu pH aumentar e ficar próximo da neutralidade (Pacifici et al., 2013; Tuloglu et al., 2014). No entanto, tem mostrado uma camada híbrida livre de hidroxiapatita, indicando que este monômero causa desmineralização da superfície dentinária, mas que a ligação química com a hidroxiapatita pode não ocorrer (Abdelraouf et al., 2019; Bumrungruan \& Sakoolnamarka, 2016; Fu et al., 2013; Poitevin et al., 2013).

Fusio Liquid Dentin tem apresentado maior resistência de união às estruturas dentárias que o Vertise Flow (Fu et al., 2013; Poitevin et al., 2013). Isso pode ser atribuído ao monômero 4-MET que apresenta valores de resistência de união maior que o GPDM às estruturas dentárias (Bumrungruan \& Sakoolnamarka, 2016). O manuseio mais fácil, o fato de ser menos pegajoso aos instrumentos de aplicação, de exibir melhor umedecimento dos tecidos dentais mineralizados e de possuir uma maior fluidez podem também contribuir em parte para a melhor adesão da Fusio Liquid Dentin em comparação com a Vertise Flow (Poitevin et al., 2013). Uma resina mais fluida favorece a penetração dos monômeros funcionais mais profundamente nas estruturas dentais, melhorando a autoadesividade (Bumrungruan \& Sakoolnamarka, 2016; Durmuşlar \& Ölmez, 2017) e a razão que explica essa diferença é que o conteúdo de carga inorgânica é menor na Fusio Liquid Dentin (65\% em peso) em comparação com Vertise Flow (70\% em peso) (Poitevin et al., 2013). Porém, ambas as resinas fluidas autoadesivas mostraram resistência ao cisalhamento inferior à resina fluida convencional (Durmuşlar \& Ölmez, 2017; Sachdeva et al., 2016; Yuan et al., 2015).

Quando aplicado em lesões cervicais não cariosas, a Fusio Liquid Dentin, apresentou uma taxa de sucesso de apenas $33 \%$, apontando falha na retenção, após 6 meses de acompanhamento contra 100\% de taxa de sucesso das resinas compostas nano-híbridas associada ao adesivo dentinário de condicionamento ácido total (Çelik et al., 2015).

Tem sido mostrado também que a aplicação prévia de algum sistema adesivo dentinário antes da resina fluida autoadesiva aumenta a resistência de união à dentina e ao esmalte (Bektas et al., 2013). A melhor combinação seria com adesivos de três passos de condicionamento ácido total do que com os adesivos autocondicionantes (Bumrungruan \& Sakoolnamarka, 2016; Goracci et al., 2013). Nos adesivos de três passos, o fosfato de cálcio, que é o produto da desmineralização do substrato dentário com ácido fosfórico, é removido após o enxágue. Já nos adesivos autocondicionantes, como não ocorre este enxágue, o fosfato de cálcio é incorporado na interface adesiva, enfraquecendo a união (Durmuşlar \& Ölmez, 2017). Porém a incorporação de mais uma etapa operatória reduz as vantagens das resinas autoadesivas, pois envolveria um cenário de manuseio mais complexo (Pacifici et al., 2013).

Apesar das fabricantes garantirem que as resinas compostas fluidas autoadesivas têm uma ampla indicação, seu uso deve ser realizado com cuidado, pelo menos até os resultados dos estudos provarem as suas vantagens clínicas sobre as resinas convencionais, tanto as fluidas (Sachdeva et al., 2016) como as de maior viscosidade (Brueckner et al., 2017).

\section{Conclusão}

De acordo com a revisão sistemática realizada pode se concluir que:

- A resina fluida autoadesiva demonstrou valores de resistência de união ao esmalte e à dentina inferior à resina fluida convencional.

- A associação do sistema adesivo de condicionamento ácido total com resina fluida autoadesiva mostrou valores de 
resistência de união maior que o uso dos autoadesivos sozinhos.

- A resina fluida autoadesiva obteve valores de resistência de união similar aos materiais restauradores à base de ionômero de vidro.

Portanto, mais estudos in vitro e in vivo são necessários para esclarecer se a resistência de união e a capacidade de vedamento das resinas compostas fluidas autoadesivas são clinicamente adequadas.

\section{Referências}

Abdelraouf, R. M., Mohammed, M., \& Abdelgawad, F. (2019). Evaluation of Shear-Bond-Strength of Dental Self-Adhering Flowable Resin-Composite versus Total-Etch One to Enamel and Dentin Surfaces: An In-Vitro Study. Open Access Macedonian Journal of Medical Sciences, 7(13), 2162-2166. https://doi.org/10.3889/oamjms.2019.579

Baroudi, K., Silikas, N., \& Watts, D. C. (2007). Time-dependent visco-elastic creep and recovery of flowable composites. European Journal of Oral Sciences, 115(6), 517-521. https://doi.org/10.1111/j.1600-0722.2007.00487.x

Bayne, S. C., Thompson, J. Y., Swift, E. J., Stamatiades, P., \& Wilkerson, M. (1998). A characterization of first-generation flowable composites. Journal of the American Dental Association (1939), 129(5), 567-577. https://doi.org/10.14219/jada.archive.1998.0274

Bektas, O. O., Eren, D., Akin, E. G., \& Akin, H. (2013). Evaluation of a self-adhering flowable composite in terms of micro-shear bond strength and microleakage. Acta Odontologica Scandinavica, 71(3-4), 541-546. https://doi.org/10.3109/00016357.2012.696697

Brueckner, C., Schneider, H., \& Haak, R. (2017). Shear Bond Strength and Tooth-Composite Interaction With Self-Adhering Flowable Composites. Operative Dentistry, 42(1), 90-100. https://doi.org/10.2341/15-365-L

Bumrungruan, C., \& Sakoolnamarka, R. (2016). Microshear bond strength to dentin of self-adhesive flowable composite compared with total-etch and all-inone adhesives. Journal of Dental Sciences, 11(4), 449-456. https://doi.org/10.1016/j.jds.2016.08.003

Çelik, E. U., Aka, B., \& Yilmaz, F. (2015). Six-month Clinical Evaluation of a Self-adhesive Flowable Composite in Noncarious Cervical Lesions. The Journal of Adhesive Dentistry, 17(4), 361-368. https://doi.org/10.3290/j.jad.a34556

Czasch, P., \& Ilie, N. (2013). In vitro comparison of mechanical properties and degree of cure of a self-adhesive and four novel flowable composites. The Journal of Adhesive Dentistry, 15(3), 229-236. https://doi.org/10.3290/j.jad.a29530

Durmuşlar, S., \& Ölmez, A. (2017). Microtensile Bond Strength and Failure Modes of Flowable Composites on Primary Dentin with Application of Different Adhesive Strategies. Contemporary Clinical Dentistry, 8(3), 373-379. https://doi.org/10.4103/ccd.ccd_310_17

ElEmbaby, A. E., Slais, M., Alawami, A., Alabbad, M., Alsowyan, M., Hanna, N., \& Bahgat, H. (2021). Spectrophotometric Analysis of Different Flowable Restorative Materials. The Journal of Contemporary Dental Practice, 22(2), 111-116.

Eliades, A., Birpou, E., Eliades, T., \& Eliades, G. (2013). Self-adhesive restoratives as pit and fissure sealants: A comparative laboratory study. Dental Materials : Official Publication of the Academy of Dental Materials, 29(7), 752-762. https://doi.org/10.1016/j.dental.2013.04.005

Fu, J., Kakuda, S., Pan, F., Hoshika, S., Ting, S., Fukuoka, A., Bao, Y., Ikeda, T., Nakaoki, Y., Selimovic, D., Sano, H., \& Sidhu, S. K. (2013). Bonding performance of a newly developed step-less all-in-one system on dentin. Dental Materials Journal, 32(2), 203-211. https://doi.org/10.4012/dmj.2012-204

Garcia, R. N., Silva, C. S., Silva, G. G., Mocellin, G., Ozelame, J., Fracasso, L., Ozelame, M. B., Nascimento, R. F. d., \& Gomes., A. C. R. (2014). Bonding performance of a self-adhering flowable composite to indirect restorative materials. Revista Sul-Brasileira De Odontologia, 11(1), 6-12.

Goracci, C., Margvelashvili, M., Giovannetti, A., Vichi, A., \& Ferrari, M. (2013). Shear bond strength of orthodontic brackets bonded with a new selfadhering flowable resin composite. Clinical Oral Investigations, 17(2), 609-617. https://doi.org/10.1007/s00784-012-0729-x

Ikeda, I., Otsuki, M., Sadr, A., Nomura, T., Kishikawa, R., \& Tagami, J. (2009). Effect of filler content of flowable composites on resin-cavity interface. Dental Materials Journal, 28(6), 679-685. https://doi.org/10.4012/dmj.28.679

Jordehi, A. Y., Shahabi, M. S., \& Akbari, A. (2019). Comparison of self-adhering flowable composite microleakage with several types of bonding agent in class V cavity restoration. Dental Research Journal, 16(4), 257-263.

Labella, R., Lambrechts, P., van Meerbeek, B [B.], \& Vanherle, G. (1999). Polymerization shrinkage and elasticity of flowable composites and filled adhesives. Dental Materials, 15(2), 128-137. https://doi.org/10.1016/S0109-5641(99)00022-6

Miletić, V. (2018). Dental Composite Materials for Direct Restorations. Springer. https://doi.org/10.1007/978-3-319-60961-4

Muñoz, M. A., Luque, I., Hass, V., Reis, A., Loguercio, A. D., \& Bombarda, N. H. C. (2013). Immediate bonding properties of universal adhesives to dentine. Journal of Dentistry, 41(5), 404-411. https://doi.org/10.1016/j.jdent.2013.03.001

Naga, A. A. E., Yousef, M., Ramadan, R., Fayez Bahgat, S., \& Alshawwa, L. (2015). Does the use of a novel self-adhesive flowable composite reduce nanoleakage? Clinical, Cosmetic and Investigational Dentistry, 7, 55-64. https://doi.org/10.2147/CCIDE.S80462 
Oz, F. D., Ergin, E., Cakir, F. Y., \& Gurgan, S. (2020). Clinical Evaluation of a Self-Adhering Flowable Resin Composite in Minimally Invasive Class I Cavities: 5-year Results of a Double Blind Randomized, Controlled Clinical Trial. Acta Stomatologica Croatica, 54(1), 10-21. https://doi.org/10.15644/asc54/1/2

Pacifici, E., Chazine, M., Vichi, A., Grandini, S., Goracci, C., \& Ferrari, M. (2013). Shear-bond strength of a new self-adhering flowable restorative material to dentin of primary molars. The Journal of Clinical Pediatric Dentistry, 38(2), 149-154. https://doi.org/10.17796/jcpd.38.2.13q513128k2870j7

Peumans, M [M.], Munck, J. de [J.], van Landuyt, K. L., Poitevin, A [A.], Lambrechts, P., \& van Meerbeek, B [B.] (2010). Eight-year clinical evaluation of a 2-step self-etch adhesive with and without selective enamel etching. Dental Materials: Official Publication of the Academy of Dental Materials, 26(12), 11761184. https://doi.org/10.1016/j.dental.2010.08.190

Poitevin, A [André], Munck, J. de [Jan], van Ende, A., Suyama, Y., Mine, A., Peumans, M [Marleen], \& van Meerbeek, B [Bart] (2013). Bonding effectiveness of self-adhesive composites to dentin and enamel. Dental Materials: Official Publication of the Academy of Dental Materials, 29(2), 221-230. https://doi.org/10.1016/j.dental.2012.10.001

Poorzandpoush, K., Shahrabi, M., Heidari, A., \& Hosseinipour, Z. S. (2019). Shear Bond Strength of Self-Adhesive Flowable Composite, Conventional Flowable Composite and Resin-Modified Glass Ionomer Cement to Primary Dentin. Frontiers in Dentistry, 16(1), 62-68. https://doi.org/10.18502/fid.v16i1.1111

Poss, S. D. (2010). Utilization of a new self-adhering flowable composite resin. Dentistry Today, 29(4), 104-105.

Sachdeva, P., Goswami, M., \& Singh, D. (2016). Comparative evaluation of shear bond strength and nanoleakage of conventional and self-adhering flowable composites to primary teeth dentin. Contemporary Clinical Dentistry, 7(3), 326-331. https://doi.org/10.4103/0976-237X.188549

Salerno, M., Derchi, G., Thorat, S., Ceseracciu, L., Ruffilli, R., \& Barone, A. C. (2011). Surface morphology and mechanical properties of new-generation flowable resin composites for dental restoration. Dental Materials: Official Publication of the Academy of Dental Materials, 27(12), 1221-1228. https://doi.org/10.1016/j.dental.2011.08.596

Shafiei, F., \& Saadat, M. (2016). Micromorphology and bond strength evaluation of adhesive interface of a self-adhering flowable composite resin-dentin: Effect of surface treatment. Microscopy Research and Technique, 79(5), 403-407. https://doi.org/10.1002/jemt.22643

Tuloglu, N., Sen Tunc, E., Ozer, S., \& Bayrak, S. (2014). Shear bond strength of self-adhering flowable composite on dentin with and without application of an adhesive system. Journal of Applied Biomaterials \& Functional Materials, 12(2), 97-101. https://doi.org/10.5301/jabfm.5000166

Unterbrink, G. L., \& Liebenberg, W. H. (1999). Flowable resin composites as "filled adhesives": Literature review and clinical recommendations. Quintessence International (Berlin, Germany: 1985), 30(4), 249-257.

van Meerbeek, B [Bart], Yoshihara, K [Kumiko], van Landuyt, K., Yoshida, Y [Yasuhiro], \& Peumans, M [Marleen] (2020). From Buonocore's Pioneering Acid-Etch Technique to Self-Adhering Restoratives. A Status Perspective of Rapidly Advancing Dental Adhesive Technology. The Journal of Adhesive Dentistry, 22(1), 7-34. https://doi.org/10.3290/j.jad.a43994

Vichi, A., Margvelashvili, M., Goracci, C., Papacchini, F., \& Ferrari, M. (2013). Bonding and sealing ability of a new self-adhering flowable composite resin in class I restorations. Clinical Oral Investigations, 17(6), 1497-1506. https://doi.org/10.1007/s00784-012-0846-6

Wei, Y., Silikas, N., Zhang, Z., \& Watts, D. C. (2011a). Diffusion and concurrent solubility of self-adhering and new resin-matrix composites during water sorption/desorption cycles. Dental Materials: Official Publication of the Academy of Dental Materials, 27(2), 197-205. https://doi.org/10.1016/j.dental.2010.10.014

Wei, Y., Silikas, N., Zhang, Z., \& Watts, D. C. (2011b). Hygroscopic dimensional changes of self-adhering and new resin-matrix composites during water sorption/desorption cycles. Dental Materials: Official Publication of the Academy of Dental Materials, 27(3), 259-266. https://doi.org/10.1016/j.dental.2010.10.015

Yoshida, Y [Y.], Nagakane, K., Fukuda, R., Nakayama, Y., Okazaki, M., Shintani, H., Inoue, S., Tagawa, Y., Suzuki, K., Munck, J. de [J.], \& van Meerbeek, B [B.] (2004). Comparative study on adhesive performance of functional monomers. Journal of Dental Research, 83(6), 454-458. https://doi.org/10.1177/154405910408300604

Yoshida, Y [Y.], Yoshihara, K [K.], Nagaoka, N., Hayakawa, S., Torii, Y., Ogawa, T., Osaka, A., \& van Meerbeek, B [B.] (2012). Self-assembled Nanolayering at the Adhesive interface. Journal of Dental Research, 91(4), 376-381. https://doi.org/10.1177/0022034512437375

Yuan, H., Li, M., Guo, B., Gao, Y., Liu, H., \& Li, J. (2015). Evaluation of Microtensile Bond Strength and Microleakage of a Self-adhering Flowable Composite. The Journal of Adhesive Dentistry, 17(6), 535-543. https://doi.org/10.3290/j.jad.a35253 